\title{
Cytogenetic Map of Pummelo and Chromosome Evolution of True Citrus Species and the Hybrid Sweet Orange
}

\author{
Silvokleio da Costa Silva ${ }^{1}$, Sandra Mendes ${ }^{1}$, Thallitha Régis ${ }^{1}$, Orlando Sampaio Passos ${ }^{2}$, \\ Walter dos Santos Soares Filho ${ }^{2} \&$ Andrea Pedrosa-Harand ${ }^{1}$ \\ ${ }^{1}$ Laboratory of Plant Cytogenetics and Evolution, Department of Botany, Federal University of Pernambuco, \\ Recife, PE, Brazil \\ 2 Embrapa Cassava \& Fruits, Cruz das Almas, BA, Brazil \\ Correspondence: Andrea Pedrosa-Harand, Laboratório de Citogenética e Evolução Vegetal, Departamento de \\ Botânica, Universidade Federal de Pernambuco, R. Prof. Moraes Rego, s/n, CDU, 50670-420, Recife, PE, Brazil. \\ Tel: 55-812-126-8846. E-mail: andrea.harand@ufpe.br
}

Received: March 31, 2019

Accepted: June 13, $2019 \quad$ Online Published: August 31, 2019

doi:10.5539/jas.v11n14p148

URL: https://doi.org/10.5539/jas.v11n14p148

The research is financed by National Council for Scientific and Technological Development (CNPq), Brazil.

\begin{abstract}
Pummelo (Citrus maxima) is considered as one of the true citrus species. Together with mandarin (C. reticulata), it gave rise to the hybrid sweet orange (C. sinensis) and other important citrus crops. Although these species have $2 n=18$, each has a unique heterochromatin distribution. The aims of this study were to identify chromosome homoeologies between pummelo and other true citrus species, to investigate the karyotypic changes involved in the chromosomal evolution between true citrus and to shed light into the origin of sweet orange hybrid karyotype. Mitotic metaphase chromosomes of pummelo and sweet orange were double stained with the fluorochromes CMA/DAPI (Chromomycin A A $_{3}$ '-6-diamidino-2-phenylindole), and identified by FISH (Fluorescence in Situ Hybridization) with chromosome-specific BAC (Bacterial Artificial Chromosome) markers. The results were compared to previously established cytogenetic maps of mandarin, C. medica and Poncirus trifoliata. Only chromosomes 1, 4 and 8 were maintained unaltered among species, with chromosomes 2 and 3 being among the least conserved in heterochromatin distribution. BACs were conserved in position among homoeologs and the markers mapped to chromosomes 2 and 3 indicated that sweet orange karyotype largely conserved one chromosome from pummelo and one from mandarin. Despite conserved synteny, expansion and contraction of heterochromatic blocks accounted for the differences between karyotypes, even between the hybrid sweet orange and pummelo.
\end{abstract}

Keywords: BAC, comparative mapping, FISH, heterochromatin, karyotype evolution, synteny

\section{Introduction}

Citrus maxima (Burm.) Merrill (Aurantioideae, Rutaceae), formerly classified as C. grandis (L.) Osbeck, and more commonly known as pummelo, is mostly cultivated in Southeast Asia for consumption as fresh fruit (Swingle \& Reece, 1967). Morphological, molecular and more recently genomic data confirmed C. maxima, as well as $C$. medica $\mathrm{L}$. and $C$. reticulata Blanco, as the main ancestral citrus species, also known as true, pure, wild or basic species. By crossing between them and other species of this genus, hybrids of economic importance were produced, such as $C$. sinensis (L.) Osbeck (sweet orange), C. aurantium (L.) (sour orange) and C. limon (L.) Burm. f. (lemon) (Scora, 1975; Barrett \& Rhodes, 1976; Nicolosi et al., 2000; Moore, 2001; Velasco \& Licciardello, 2014; Wu et al., 2014; Wu et al., 2018).

Pummelo, similarly to the other Citrus species and related genera, shows a very stable chromosome number ( $2 n$ $=2 x=18$ ), with small chromosomes ( 2 to $4 \mu \mathrm{m})$, meta- to submetacentric. Nevertheless, fluorochrome staining using chromomycin $\mathrm{A}_{3}$ (CMA) and 4'-6-diamidino-2-phenylindole (DAPI) revealed different banding patterns in Citrus, facilitating the characterization of karyotypes (Guerra, 2009). According to the distribution pattern of the $\mathrm{CMA}^{+} / \mathrm{DAPI}^{-}$heterochromatic bands, various chromosomal types have been distinguished in the genus: A (two terminal and one proximal band), B (one terminal and one proximal band), C (two terminal bands), D (one 
terminal band), $\mathrm{E}$ (one interstitial band), F (no band) and $\mathrm{G}$ (two bands localized in the same chromosomal arm, one subterminal and another terminal). The $\mathrm{F}_{\mathrm{L}}$ type is the largest among the $\mathrm{F}$ type chromosomes (Guerra, 1993; Carvalho et al., 2005). All karyotypes of the Citrus accessions studied revealed the presence of D and F type chromosomes, while types $\mathrm{A}, \mathrm{B}, \mathrm{C}, \mathrm{E}$ and $\mathrm{G}$ are usually rare and are utilized as chromosome markers to differentiate among the accessions of this genus (Cornélio et al., 2003; Carvalho et al., 2005; Moraes et al., 2007a, 2007b; Guerra, 2009), and others related to it (Brasileiro-Vidal et al., 2007). Pure species show homomorphic karyotypes (both homoeologs with similar morphology and banding pattern), while hybrid accessions show heteromorphic karyotypes (Guerra, 2009).

'Pink' and 'Israel' pummelo cultivars revealed a homomorphic karyotype formula with $4 \mathrm{~A}+2 \mathrm{C}+4 \mathrm{D}+6 \mathrm{~F}+2 \mathrm{~F}_{\mathrm{L}}$ (Moraes et al., 2007b; Barros e Silva et al., 2010), supporting pummelo as a pure Citrus species. Previous reports had proposed slightly different karyotype formulae for certain C. maxima accessions, with heteromorphism in at least one of their chromosome pairs (Guerra, 1993; Befu et al., 2000; Yang et al., 2002; Yamamoto et al., 2007). These differences may suggest a hybrid origin for some analysed materials, but they may be, at least in part, due to technical limitations, since some A chromosomes have faint terminal bands (therefore not detected in all cells) due to the low amount of CsSat, the satellite DNA sequence present in these $\mathrm{CMA}^{+}$bands (Guerra, 2009; Barros e Silva et al., 2010). A combined analysis of the $\mathrm{CMA}^{+} / \mathrm{DAPI}^{-}$heterochromatic banding pattern, as well as the distribution of rDNA (ribosomal DNA) sites using fluorescence in situ hybridization (FISH), enabled the identification of the type A chromosomes and a pair of $\mathrm{F}$ chromosomes as carriers of the $35 \mathrm{~S}$ (also known as 45S) and $5 \mathrm{~S}$ rDNA sites, respectively (A/35S and F/5S) in pummelo (Moraes et al., 2007b). The F/5S subtype chromosome is unique to C. maxima and its hybrids and was observed in 'Orlando' tangelo (C. paradisi Macfad. $\times$ C. tangerina hort. ex Tanaka), pummelo and sweet orange (Pedrosa et al., 2000; Moraes et al., 2007a, 2007b).

Among the species included in this genus, $C$. sinensis (sweet orange) is the most widely cultivated. Its cytogenetic analysis revealed that, although this species has a heteromorphic karyotype $(2 \mathrm{~B}+2 \mathrm{C}+7 \mathrm{D}+5 \mathrm{~F}+$ $2 \mathrm{~F}_{\mathrm{L}}$ ) due to its hybrid origin, all analysed cultivars have the same karyotype, probably because of its asexual propagation (Matsuyama et al., 1996; Miranda et al., 1997; Befu et al., 2000; Pedrosa et al., 2000). Indeed ten cultivars of sweet orange showed they were all derived from the same genome by somatic mutations (Wu et al., 2018). Due to its heteromorphic karyotype, chromosome pairs could not be recognized and its 18 chromosomes were separated in four groups according to their $\mathrm{CMA}^{+} / \mathrm{DAPI} b a n d s$. FISH with rDNA probes revealed two $\mathrm{B} / 35 \mathrm{~S}$,one $\mathrm{D} / 5 \mathrm{~S}-35 \mathrm{~S}$ and one F/5S (Pedrosa et al., 2000). Correlation between chromosomes and genome scaffolds is still not available (Xu et al., 2013).

Although different studies confirm C. maxima and C. reticulata as C. sinensis parents (Scora, 1975; Barrett \& Rhodes, 1976; Green et al., 1986; Yamamoto et al., 1993; Nicolosi et al., 2000; Moore, 2001; Barkley et al., 2006; Uzun et al., 2009; Li et al., 2010; Froelicher et al., 2011), its exact origin remains controversial (Xu et al., 2013; Wu et al., 2014), because its karyotype is not as expected from a simple hybridization scheme (Pedrosa et al., 2000; Guerra, 2009) and its genome constitution reveals a complex history of admixture (Wu et al., 2018). However, as its chromosomes could not be individually distinguished, the degree to which the parental chromosome types are present in this hybrid could not be confirmed. Nevertheless, the combination of $\mathrm{CMA}^{+} / \mathrm{DAPI}^{-}$banding, location of the rDNA sites and BAC-FISH in Poncirus trifoliata (L.) Raf. (4B + 8D $+4 \mathrm{~F}$ $+2 \mathrm{~F}_{\mathrm{L}}$ ), a close relative of Citrus, enabled the identification of the nine chromosome pairs of the species (Moraes et al., 2008; Da Costa Silva et al., 2011). Using these markers, comparative cytogenetic maps of $C$. medica 'Etrog' (Mendes et al., 2011) and C. reticulata 'Cravo' (Da Costa Silva et al., 2015) were constructed. Considering the available markers, the chromosomes pairs 2 and 3 showed the most polymorphism among the species studied.

The major aim of this study was to build a comparative cytogenetic map of pummelo, finishing the mapping of the three main ancestral citrus species: citron (Mendes et al., 2011), mandarin (Da Costa Silva et al., 2015) and pummelo (present work). Besides, we analyzed the chromosomes 2 and 3 in sweet orange to examine the putative karyotypic changes involved in the origin of this hybrid species.

\section{Materials and Methods}

\subsection{Plant Material and Chromosome Preparations}

Citrus maxima 'Siamese Pink' (further referred to as 'Pink') and C. sinensis 'Valencia' seeds were obtained from the Citrus Active Germplasm Bank of Embrapa Cassava \& Fruits, Cruz das Almas, Bahia, Brazil. 'Pink' is the male parent of 'Chandler', which has low-acid pummelo, 'Siamese sweet', as female parent (both recently sequenced, Wu et al., 2014). 'Pink' was selected because it is the male parent of 'Chandler', already sequenced, and it has a well-characterized, homomorphic karyotype, excluding it as a possible hybrid (Moraes et al., 2007b). 
Its seeds were introduced in Brazil from the UCR Citrus Variety Collection- University of California, Riverside. Three grafted individuals are being maintained near to 'Israel' and 'Chandler' pummelos. Because pummelo is self-incompatible and its seeds are monoembryonic, it is expected that seedlings are derived from crosses between different individuals. Therefore, we selected for further analysis only seedlings that showed after CMA/DAPI staining (see below) the same homomorphic karyotype already described for the mother plant by Moraes et al. (2007b). 'Valencia' is one of the sweet orange leading varieties worldwide and it has recently been sequenced as a dihaploid line (Xu et al., 2013). Although previous studies suggested it has a reciprocal translocation (Lan et al., 2016), no evidence for such translocation was obtained from cytogenetic (Pedrosa et al., 2000) or genomic analyses (Wu et al., 2014, 2018).

For cytogenetic analyses, root tips from the germinated seeds were pre-treated with 8-hydroxyquinoline $(0.002$ M) for $5 \mathrm{~h}$ at $18{ }^{\circ} \mathrm{C}$. They were fixed in a ratio of 3:1 (v/v) ethanol/acetic acid for $18-24 \mathrm{~h}$ at room temperature and stored at $-20^{\circ} \mathrm{C}$. The cytological preparations were performed according to Da Costa Silva et al. (2011).

\subsection{CMA/DAPI Staining}

After aging for three days, the slides were stained with CMA and DAPI according to Moraes et al. (2007a), with $0.1 \mathrm{mg} / \mathrm{ml} \mathrm{CMA}$. The slides were mounted in Mcllvaine's buffer (pH 7.0) and glycerol $(1: 1, \mathrm{v} / \mathrm{v})$ and the best metaphases were photographed after three days, using an epifluorescence Leica DMLB microscope, equipped with a Cohu CCD video camera, employing the QFISH Leica software. Slides from seedlings showing the maternal karyotype formula were destained before storing at $-20{ }^{\circ} \mathrm{C}$ for later use for in situ hybridization.

\subsection{Probes}

Nine BACs (01B09, 02C12, 20C13, 14A12, 21L13, 24C13, 28A05, 28A07 and 59C23), previously established as chromosomal markers (Moraes et al., 2008; Da Costa Silva et al., 2011) were chosen from the P. trifoliata 'Pomeroy' genomic library (Yang et al., 2001) and used for BAC-FISH in C. maxima. The 5S rDNA sites were located using the D2 plasmid clone from Lotus japonicus (Pedrosa et al., 2002). Markers of chromosomes 2 (BAC 21L13) and 3 (28A07) were used to identify these chromosomes in C. sinensis. Isolation of the DNA of BACs and plasmid was done employing the Plasmid Mini Kit (Qiagen) according to the manufacturer's protocol Labelling of all the probes was accomplished using the Nick Translation Mix (Roche Diagnostics) with Cy3-dUTP (GE).

\subsection{Fluorescence in Situ Hybridization (FISH)}

The FISH procedure was performed based on the protocol of Da Costa Silva et al. (2011), with $72 \%$ final stringency. Chromosomes were counterstaining with $2 \mu \mathrm{g} / \mathrm{ml}$ DAPI in Vectashield medium (Vector). Rehybridization was performed to detect different DNA sequences in the same cell, according to Heslop-Harrison et al. (1992). The images were superimposed and adjusted for brightness and contrast using Adobe Photoshop CS3 version 10.0.

\subsection{Measurements of Chromosomes}

The mean relative chromosome size, the size of the $\mathrm{CMA}^{+}$band and arm ratios were calculated for each chromosome pair of C. maxima based on measuring five mitotic metaphases, according to Da Costa Silva et al. (2011). The absolute chromosome sizes were estimated in megabase pairs (Mbp) based on the genome size $(0.779 \mathrm{pg} / 2 \mathrm{C})$ estimated for 'Pink' by Ollitrault et al. (1994). Chromosome morphology classification followed Guerra (1986) and chromosome types, Carvalho et al. (2005).

The software Image Tool 3.0 was used to locate the relative position of single copy BACs in C. maxima according to Fonsêca et al. (2010) and Da Costa Silva et al. (2011). Measurements were performed in 15 chromatids per BAC clone. Chromosomes were numbered according to the nomenclature proposed by Da Costa Silva et al. (2011). Relative position of BACs in relation to chromosome types in C. sinensis was confirmed in at least five metaphases.

\section{Results}

\subsection{Citrus maxima 'Pink'}

The genome size of C. maxima 'Pink' is approximately $380.9 \mathrm{Mbp}(1 \mathrm{C}=0.39 \mathrm{pg}$, as established by Ollitrault et al., 1994). It is distributed on nine chromosomes $(2 n=18)$, which are either meta- or submetacentric, with sizes ranging from 1.69 to 2.52 micrometers (Figure 1 and Figure A1). According to the number and distribution of $\mathrm{CMA}^{+} / \mathrm{DAPI}^{-}$heterochromatic bands, the karyotype formula was $4 \mathrm{~A}+2 \mathrm{C}+4 \mathrm{D}+6 \mathrm{~F}+2 \mathrm{~F}_{\mathrm{L}}$. As previously shown (Guerra, 2009), even in individuals showing this karyotype formula in most cells, in some metaphases the 
small terminal $\mathrm{CMA}^{+} / \mathrm{DAPI}^{-}$band in the short arm of one type A pair (Figure 1a) or the faint proximal band of the other A pair were not observed (Figure 1c), resembling types B and C chromosomes, respectively.
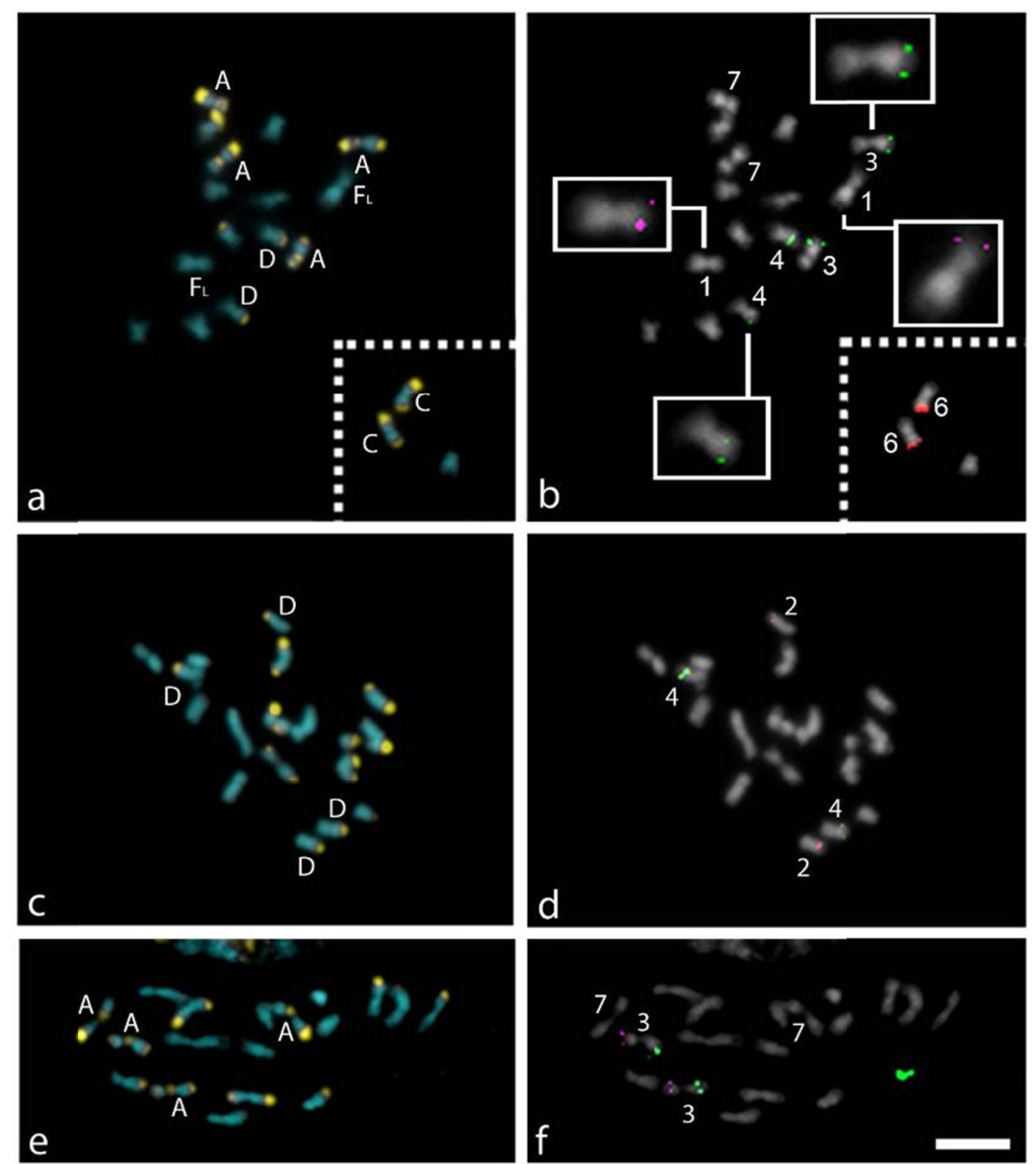

Figure 1. Mitotic metaphases of Citrus maxima (Burm.) Merril 'Pink' stained with CMA/DAPI (a, c and e) and in situ hybridized with BACs 24C13 (pink, chr. 1), 28A05 (red, chr. 6) (b) 20C13 (green, chr. 4) (b and d), 28A07 (green, chr. 3) (b and f), $21 \mathrm{~L} 13$ (red, chr. 2) (d) and 14A12 (pink, chr. 3) (f). Chromosomes 7 were identified as type A without BAC signal (b and $\mathrm{f}$ ). The dashed insert in (a) and (b) indicates three chromosomes of the same metaphase that were approximated. Insets in (b) show enlarged chromosomes 1, 3 and 4. Bar in (f) corresponds to 2.5 micrometers

Employing a set of nine BAC probes and the $5 \mathrm{~S}$ rDNA, in association with the $\mathrm{CMA}^{+} / \mathrm{DAPI}^{-}$banding pattern, all chromosome pairs of the complement were identified and classified based on the citrus nomenclature (Da Costa Silva et al., 2011), and confirmed 'Pink' as a homomorphic accession. Measurements of the $\mathrm{CMA}^{+}$band sizes revealed that about $23 \%$ of the $C$. maxima 'Pink' karyotype is composed of $\mathrm{CMA}^{+} / \mathrm{DAPI}^{-}$constitutive heterochromatin, ranging from $23.7 \%$ in chromosome 4 to $55.2 \%$ in chromosome 3 . However, no heterochromatic blocks were visible in chromosomes 1, 5, 8 and 9 (Figure A1).

Chromosome 1, type $\mathrm{F}_{\mathrm{L}}$, metacentric, was identified by the hybridization of BAC $24 \mathrm{C} 13$ in the terminal region of its short arm (Figures 1a-1b). The BAC $21 \mathrm{~L} 13$ hybridized adjacently to the $\mathrm{CMA}^{+} / \mathrm{DAPI}^{-}$band of the long arm of chromosome 2 (type D, metacentric) (Figures 1c-1d).

Chromosomes 3 (type A, metacentric) and 4 (type D, metacentric) were identified by two BACs each. In chromosome 3, the BACs 14A12 and 28A07 were detected adjacent to the $\mathrm{CMA}^{+} / \mathrm{DAPI}$ bands on the short and long arms, respectively (Figures 1e-1f). In this chromosome, the proximal $\mathrm{CMA}^{+}$band was not always clearly visible. Chromosome 4 was identified by BACs $02 \mathrm{C} 12$ (data not shown) and 20C13 (Figures 1c-1d) mapped in the interstitial region of the short arm and adjacent to the heterochromatic band of the long arm, respectively. 
Chromosome 5 (a submetacentric type F) was identified by the $5 \mathrm{~S}$ rDNA site in the proximal region of the short arm (Figures A2a-A2b). In one of the six individuals analysed with the 5S rDNA probe, signals were observed in a heteromorphic pair: in one type $\mathrm{F}$ chromosome and adjacent to the $\mathrm{CMA}^{+} / \mathrm{DAPI}{ }^{-}$band of a type $\mathrm{D}$ chromosome (data not shown). Since the BAC 28A05 hybridized adjacent to the $\mathrm{CMA}^{+} / \mathrm{DAPI}$ band of the short arm of the type $\mathrm{C}$ pair (metacentric), it was identified as chromosome 6 (Figures 1a-1b). The submetacentric chromosomes 8 and 9 were both type F. The BAC 01B09 was mapped in the terminal region of the short arm of chromosome 8 (data not shown), whereas the presence of the BAC 59C23, associated with resistance to the citrus tristeza virus (CTV) (Yang et al., 2001), in the interstitial region of the long arm enabled the recognition of the chromosome 9 pair (Figures A2c-A2d).

No BAC marker has been described for chromosome 7 (submetacentric type A). In the metaphases where type A chromosome 3 was detected by markers, the terminal $\mathrm{CMA}^{+} / \mathrm{DAPI}^{-}$band in the short arm of chromosome 7 is normally smaller than from chromosome 3, and sometimes not visible (Figures 1a and 1e). The relative position of each marker is shown in the idiogram (Figure A1).

\subsection{Chromosome Markers in Citrus sinensis 'Valencia'}

The karyotype formula $2 \mathrm{~B}+2 \mathrm{C}+7 \mathrm{D}+5 \mathrm{~F}+2 \mathrm{~F}_{\mathrm{L}}$ (Figure 2a) was confirmed for $C$. sinensis. BAC markers for chromosomes 2 and 3, which differ in respect to the $\mathrm{CMA}^{+} / \mathrm{DAPI}^{-}$banding pattern between both parents (pummelo and mandarin), were used to analyse these chromosomes in the hybrid sweet orange. Chromosome 2 pair, recognized by BAC 21L13, was indeed heteromorphic in C. sinensis. Although both were type D, in one homoeolog the signal was observed in the terminal region of the short arm and in the other homoeolog it was adjacent to a smaller $\mathrm{CMA}^{+} / \mathrm{DAPI}^{-}$band in the long arm (Figures 2a-2b), as expected from mandarin and pummelo chromosomes, respectively.
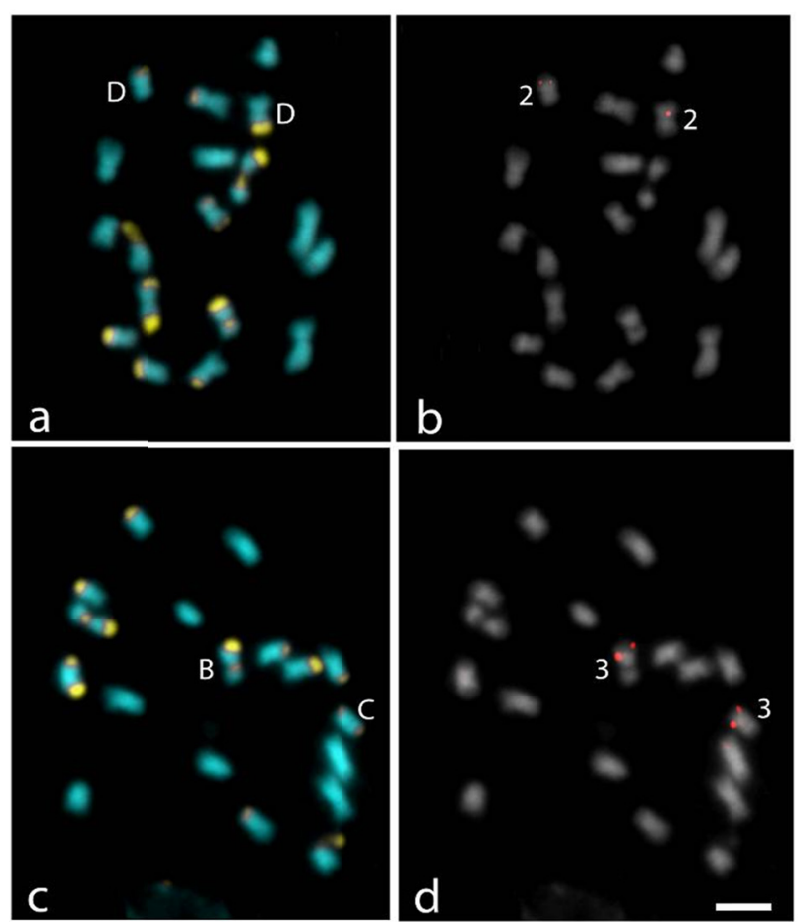

Figure 2. Mitotic metaphases of Citrus sinensis (L.) Osbeck 'Valencia' stained with CMA/DAPI (a and c) and in situ hybridization with BACs $21 \mathrm{~L} 13$ (red, chr. 2) (b) and 28A07 (red, chr. 3) (d). Bar in (d) corresponds to 2.5 micrometers

The presence of BAC 28A07 enabled the identification of the chromosome pair 3 in sweet orange, which was also heteromorphic. This BAC was mapped adjacent to the $\mathrm{CMA}^{+} / \mathrm{DAPI}^{-}$bands in the long arms of a type $\mathrm{B}$ and in the smallest type $\mathrm{C}$ chromosome (Figures $2 \mathrm{c}-2 \mathrm{~d}$ ). Thus, as expected from the parental karyotypes, this probe confirmed that neither type $\mathrm{B}$ or $\mathrm{C}$ chromosomes are homoeologous in sweet orange (Figure 3 ). 

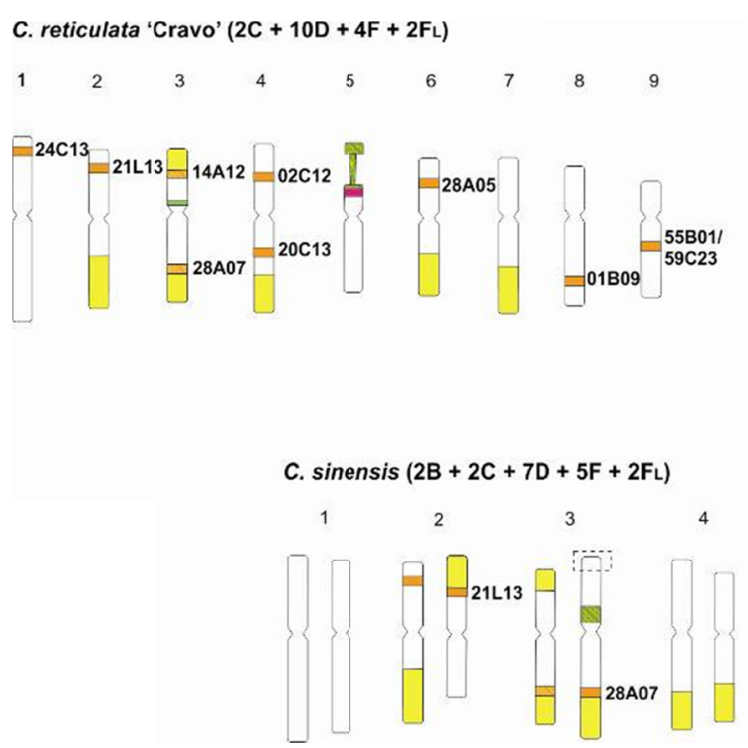
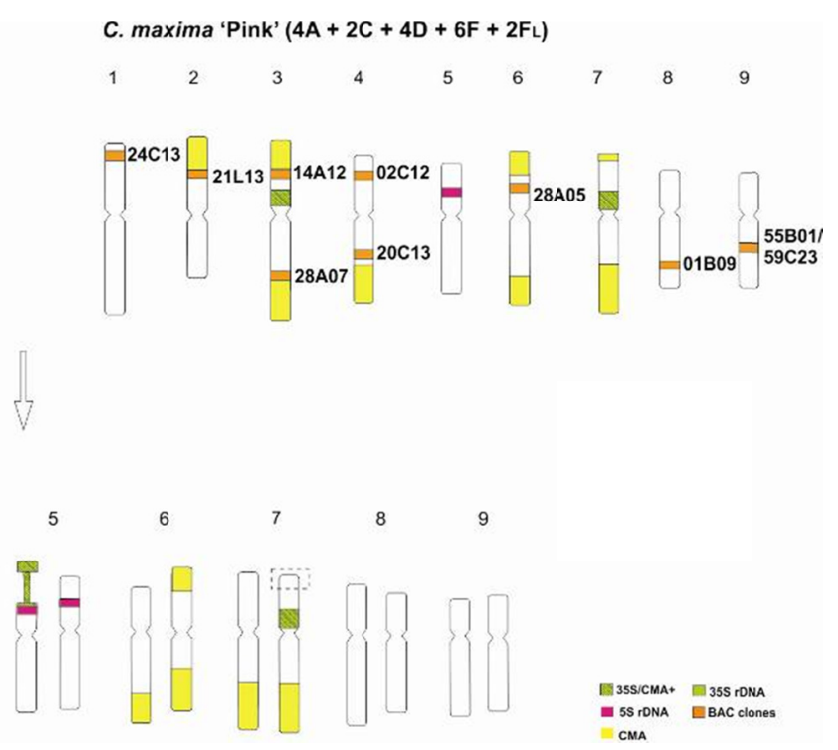

Figure 3. Schematic representation of gametic karyotypes generated by Citrus maxima (Burm.) Merril and $C$. reticulata Blanco compared with the somatic karyotype of C. sinensis (L.) Osbeck (adapted from Pedrosa et al., 2000; Da Costa Silva et al., 2015; and present work). Some chromosomes are represented upside down from the original idiogram to maintain the collinearity of BAC markers. Changes in $C$. sinensis chromosome types in relation to the expected from the parental gametes (lack of terminals $\mathrm{CMA}^{+} / \mathrm{DAPI}^{-}$bands in chromosomes 3 and

7 , resulting in type B chromosomes) are indicated by dashed squares

\section{Discussion}

A comparative cytogenetic map for pummelo 'Pink' was constructed combining the in situ hybridization of nine BAC clones established as chromosome markers for citrus (Moraes et al., 2008; Da Costa Silva et al., 2011), with the localization of $\mathrm{CMA}^{+} / \mathrm{DAPI}^{-}$heterochromatic bands and the 5S rDNA site. The cytogenetic markers utilized confirmed the homomorphic karyotype of this accession, because all BACs hybridized to a pair of chromosomes of the same type in the same position (Moraes et al., 2007b; Barros e Silva et al., 2010).

The chromosomal analysis revealed that the $\sim 380.9 \mathrm{Mbp}$ C. maxima 'Pink' genome comprises about $23.3 \%$ of $\mathrm{CMA}^{+} / \mathrm{DAPI}{ }^{-}$heterochromatin (HC). This amount of heterochromatin is slightly more than that found in other citrus species examined like P. trifoliata $(\sim 376.5 \mathrm{Mpb} ; \mathrm{HC}=21.8 \%)$, C. medica $(401.5 \mathrm{Mpb} ; \mathrm{HC}=18.4 \%)$ and C. reticulata ( $\sim 373.0$ MPB; HC $=21.4 \%$ ) (Kayum et al., 1998; Da Costa Silva et al., 2011; Mendes et al., 2011; Da Costa Silva et al., 2015), and it is compatible with the karyotypic differences in their formulae, as C. maxima is the only species with three chromosome pairs having heterochromatic bands in both arms. These differences should be associated with the contraction and/or expansion of CsSat satellite DNA, the repetitive DNA sequences that constitute most of the $\mathrm{CMA}^{+} / \mathrm{DAPI}^{-}$bands in Citrus (Barros e Silva et al., 2010).

Chromosome type A is a characteristic marker for pummelo karyotypes (Guerra, 2009). 'Pink' and 'Israel' karyotypes have four type A chromosomes (Moraes et al., 2007b; Barros e Silva et al., 2010), but different accessions previously analysed showed from one to four chromosomes A per karyotype (Miranda et al., 1997; Befu et al., 2000, 2001, 2002; Yang et al., 2002; Yamamoto et al., 2007), remaining unclear whether an intraspecific variation is present or whether these differences are a result of the differences in the sensitivity of the techniques employed (Guerra, 2009). When BAC markers for chromosome 3 were employed, a difference in the size of the heterochromatic bands between chromosomes 3 and 7 (type A) of C. maxima 'Pink' was identified. A smaller amount of CsSat is likely present in the weaker band of the short arm of chromosome 7 and is therefore not always visible as a $\mathrm{CMA}^{+}$band because of the reduced sensitivity of the CMA staining in comparison to FISH (Barros e Silva et al., 2010). Similarly to type B chromosomes (Pedrosa et al., 2000), type A chromosomes (pairs 3 and 7 of $C$. maxima) showed 35S rDNA sites co-localized with the proximal $\mathrm{CMA}^{+} / \mathrm{DAPI}^{-}$ bands and are recognized as subtype A/35S (Moraes et al., 2007a, 2007b; Barros e Silva et al., 2010). This proximal band is also evident in some cells as $\mathrm{CMA}^{0}$ (neutral for CMA staining), resulting in chromosomes that resemble a $\mathrm{C}$ type, most likely due to the small size of this rDNA site or its epigenetic status (Da Costa Silva et al., 2015). 
Pummelo also has a chromosome pair of the F/5S subtype. This subtype was detected in the chromosomal accessions derived from C. maxima, like the hybrids 'Orlando' tangelo, 'Murcott' tangor, grapefruit (C. paradisi) and sweet orange. These accessions are all derived from C. maxima crosses and have a single $\mathrm{F} / 5 \mathrm{~S}$ chromosome (Pedrosa et al., 2000; Moraes et al., 2007a, 2007b). One pummelo seedling analysed in the present study showed a $5 \mathrm{~S}$ rDNA site in one type D chromosome, what was unexpected. This could suggest a hybrid origin for this individual seedling (Moore, 2001); however, no known accession would provide two type A and one D/5S chromosome in its gamete (Guerra, 2009). Therefore, it is more likely that a rearrangement between one of its $\mathrm{F}$ and a chromosome $\mathrm{D}$ took place in this individual, changing the heterochromatin content of two chromosomes without changing its karyotype formula. A translocation event involving a $35 \mathrm{~S}$ rDNA site has been reported in the cultivars 'USDA' and 'Pomeroy' of trifoliate orange (Barros e Silva et al., 2010).

Phylogenetic studies including true and hybrid species and using different molecular markers indicated a greater proximity between $C$. maxima and $C$. reticulata than $C$. medica, and $P$. trifoliata as the most distant species (Nicolosi et al., 2000; Barkley et al., 2006; Pang et al., 2007; Uzun et al., 2009). However, the phylogenetic analysis of 27 nuclear genes, including the possible true species but excluding the hybrids, showed that $C$. maxima is more closely related to C. medica, while C. reticulata is closer to P. trifoliata (Garcia-Lor et al., 2013). The close proximity between Poncirus and Citrus was also evidenced in phylogenetic studies using chloroplast DNA sequences (Bayer et al., 2009; Penjor et al., 2010, 2013; Carbonell-Caballero et al., 2015). Recent whole-genome phylogenetic reconstructions confirmed the partial incongruence between nuclear versus plastid genome-derived phylogenies, but also confirmed the closer proximity of C. maxima and C. medica, as well as $P$. trifoliata as a distinct, early divergent clade (Wang et al., 2017; Wu et al., 2018). On comparing the cytogenetic maps of $C$. reticulata, $C$. medica and $P$. trifoliata, several changes in the $C$. medica lineage were suggested, with a relative conservation of the $C$. reticulata and $P$. trifoliata karyotypes (Mendes et al., 2011; Da Costa Silva et al., 2011; Da Costa Silva et al., 2015). The C. maxima map in fact highlights karyotype similarities with C. medica, especially on chromosome 2,3 and 5 , and confirms the greatest karyotype similarity reported between $C$. reticulata and $P$. trifoliata, representing ancestral karyotype features (Figure 4). Therefore, the chromosome data presented here support the phylogenetic relationships proposed by Wu et al. (2018). 


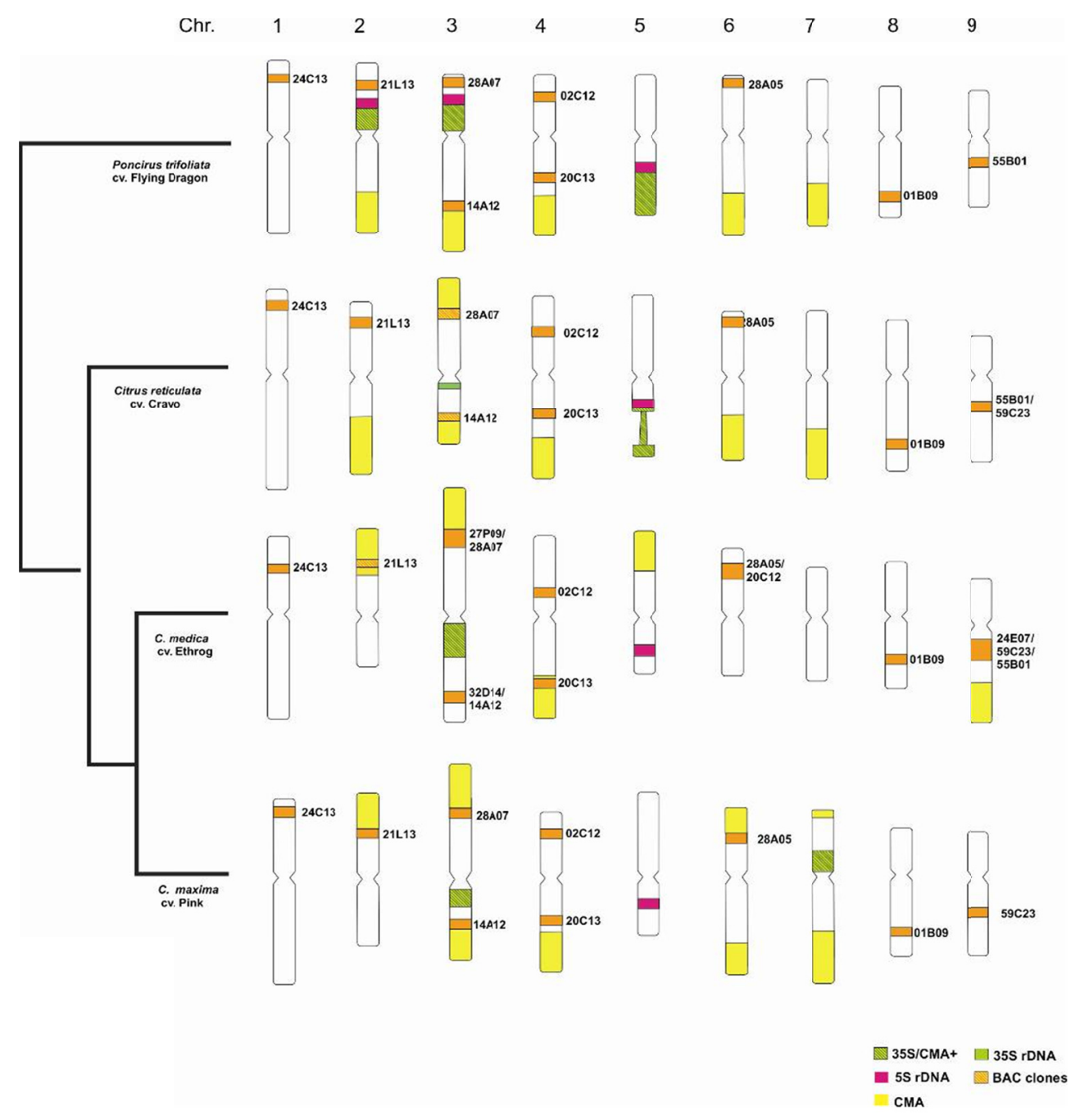

Figure 4. Schematic representation of the karyotypes of Citrus maxima (Burm.) Merril (present work) compared to C. reticulata Blanco, C. medica L. and Poncirus trifoliata (L.) Raf. (adapted from Mendes et al., 2011; Da Costa Silva et al., 2011; Da Costa Silva et al., 2015), showing the differences between chromosome types, size of CMA-positive heterochromatic bands, distribution of rDNA and BACs. Phylogenetic relationships among species were based on Wu et al. (2018). Some chromosomes are represented upside down from the original idiogram to maintain the collinearity of the BAC markers

The C. maxima map confirms that the changes in the number and position of the $\mathrm{CMA}^{+} / \mathrm{DAPI}^{-}$bands occurred often in this group. Chromosomes 1 ( $\mathrm{F}_{\mathrm{L}}$ type), 4 (D type) and 8 (F type) are the only ones that appear to remain unchanged. Chromosomes 6 and 7 experienced the expansion of heterochromatic blocks in C. maxima, while chromosomes 5 and 9 , and 6 and 7 were subjected to expansion and contraction, respectively, in the $C$. medica lineage. Chromosome 3 is perhaps the most polymorphic among species (A, B or C type). The presence of the proximal 35S rDNA site in chromosome 3 is ancestral and conserved in the group, but this site was largely reduced in size in C. reticulata (Da Costa Silva et al., 2015). Furthermore, its position was changed between chromosome arms in the Poncirus or Citrus lineages. A second ancestral rDNA site in chromosome 5, linked to a 5S rDNA site in C. reticulata and P. trifoliata (Barros e Silva et al., 2013), was lost in the C. maxima/C. medica lineage. Conservation of BAC positions indicates, however, that major translocations were never fixed in any lineage, implying a high degree of conservation of synteny within the group, as also indicated from genomic data (Wu et al., 2014).

Several lines of evidence support that $C$. sinensis has originated by hybridization of C. maxima and C. reticulata, but the exact origin of this hybrid was controversial (Scora, 1975; Barrett \& Rhodes, 1976; Green et al., 1986; Yamamoto et al., 1993; Nicolosi et al., 2000; Moore, 2001; Barkley et al., 2006; Uzun et al., 2009; Li et al., 2010; Froelicher et al., 2011; Garcia-Lor et al., 2013; Xu et al., 2013; Wu et al., 2014). Sweet orange is a hybrid of 
complex origin, because two pummelo and two mandarin alleles were observed in different chromosome segments, ruling it out as a $\mathrm{F}_{1}$ or $\mathrm{BC}_{1}$ hybrid (Garcia-Lor et al., 2013; Wu et al., 2014). After multiple introgressions, a single hybrid was probably propagated asexually and experienced genetic mutations, giving rise to the various sweet orange cultivars (Barrett \& Rhodes, 1976; Guerra, 2009; Wu et al., 2018). Based on the identification of homoeologous chromosome pairs from C. reticulata and C. maxima (Da Costa Silva et al., 2015; and present paper), it is possible to predict the parental contributions to the sweet orange karyotype as follows: (i) C. reticulata - $1 \mathrm{C}$ [Chromosome 3] $+5 \mathrm{D}\left[2,4,5,6\right.$ and 7] $+2 \mathrm{~F}$ [8 and 9] $+1 \mathrm{~F}_{\mathrm{L}}[1]$; and (ii) C. maxima-2A [3 and 7] $+1 \mathrm{C}[6]+2 \mathrm{D}[2$ and 4$]+3 \mathrm{~F}\left[5,8\right.$ and 9] $+1 \mathrm{~F}_{\mathrm{L}}$ [1] (Figure 3). But because it has a complex admixture history, sweet orange could have, for each chromosome pair, a mandarin/mandarin, mandarin/pummelo or pummelo/pummelo combination, as well as recombinant types. Our chromosomal data is, however, compatible with a sweet orange karyotype formed by one chromosome set from pummelo and one from mandarin, which have experienced previous segmental introgressions. C. reticulata 'Cravo' karyotype formula is compatible with the putative ancestral mandarin karyotype that gave rise to sweet orange (Da Costa Silva et al., 2015; present data). Sweet orange chromosome pair 2 (both $\mathrm{D}$ type) is formed by a C. reticulata chromosome, whose BAC was mapped terminally in the short arm, and the C. maxima homoeolog, whose BAC was mapped in the terminal region of the long arm. The difference between both homeologs is the presence of a heterochromatic block opposite or adjacent, respectively, to the BAC clone, changing chromosome morphology. Chromosome pair 3 was formed by one of the $\mathrm{C}$ chromosomes (originating from $C$. reticulata) and one of the Bs (probably derived from the A type C. maxima chromosome 3). Although scattered discrepancies between the two genome assemblies ('Valencia' and 'Clementina') has been observed, no large scale chromosomal reciprocal translocation was detected (Wu et al., 2014). Indeed, the karyotype of the sequenced 'Valencia' dihaploid (Xu et al., 2013; Lan et al., 2016) can be inferred as: chr. 1-type F, chr. 2-D, chr. 3-B, chr. 4-D, chr. 5-D, chr. 6-C, chr. 7-B, chr. 8-F and chr. 9-F.

As a pummelo hybrid, it was expected that two type A chromosomes (one copy of chromosome 3 and one copy of 7) had been passed down to sweet orange (Guerra, 2009). However, the presence of two type B chromosomes, not present in either parent, is observed in all sweet orange accessions (Matsuyama et al., 1996; Miranda et al., 1997; Befu et al., 2000; Pedrosa et al., 2000; Barros e Silva et al., 2010; and in this study). Some C. maxima A chromosomes (chromosome 7) are observed as Bs in different cells because of the small size of the $\mathrm{CMA}^{+}$ terminal band in the short arm (e.g., Figures 1a or 1e). In light of the high variability in the $\mathrm{CMA}^{+}$bands in Citrus, it seems possible that A chromosomes have experienced loss or gain of repetitive DNA sequences that compose these terminal bands in the ancestral or present-day pummelo accessions, respectively, or loss during sweet orange hybrid formation. Comparison of the cytogenetic maps of C. maxima, C. reticulata, C. medica and P. trifoliata showed that chromosomes 3 and 7 are the most variable among species in terms of heterochromatin distribution (Mendes et al., 2011; Da Costa Silva et al., 2011; Da Costa Silva et al., 2015; present work). The other possibility, that the B chromosomes may have been inherited from another species, not C. maxima, involved in this cross, is, however, very unlikely (Guerra, 2009; Wu et al., 2014, 2018).

\section{Conclusions}

With the constructed cytogenetic map of pummelo, the maps of the three main pure citrus species are now available. The established chromosome homeologies confirmed that the differences among pure species are related to variation in heterochromatin distribution in some chromosome pairs, mainly in chromosomes 2 and 3 . These marker chromosomes can be used to investigate chromosome composition and evolution of citrus hybrids, such as demonstrated for the main hybrid sweet orange, providing important information for the taxonomy, evolution and genetic improvement of this group.

\section{Acknowledgements}

The authors are grateful to Mr. Getúlio de Souza Vieira (Embrapa Cassava \& Fruits) for the field support and the Conselho Nacional de Desenvolvimento Científico e Tecnológico (CNPq), Brazil, for the financial support.

\section{References}

Barkley, N. A., Roose, M. L., Krueger, R. R., \& Federici, C. T. (2006). Assessing genetic diversity and population structure in the citrus germplasm collection utilizing simple sequence repeat markers (SSRs). Theoretical and Applied Genetics, 112(8), 1519-1531. https://doi.org/10.1007/s00122-006-0255-9

Barrett, H. C., \& Rhodes, A. M. (1976). A numerical taxonomic study of affinity relationships in cultivated Citrus and its close relatives. Systematic Botany, 1(2), 105-136. https://doi.org/10.2307/2418763 
Barros e Silva, A. E., Marques, A., Santos, K. G. B., \& Guerra, M. (2010). The evolution of CMA bands in Citrus and related genera. Chromosome Research, 18(4), 503-514. https://doi.org/10.1007/s10577010-9130-2

Barros e Silva, A. E., Soares Filho, W. S., \& Guerra, M. (2013). Linked 5S and 45S rDNA sites are highly conserved through the subfamily Aurantioideae (Rutaceae). Cytogenetic and Genome Research, 140(1), 62-69. https://doi.org/10.1159/000350695

Bayer, R. J., Mabberley, D. J., Morton, C., Miller, C. H., Sharma, I. K., Pfeil, B. E., ... Sykes, S. (2009). A molecular phylogeny of the orange subfamily (Rutaceae: Aurantioideae) using nine cpDNA sequences. American Journal of Botany, 96(3), 668-685. https://doi.org/10.3732/ajb.0800341

Befu, M., Kitajima, A., \& Hasegawa, K. (2001). Chromosome composition of some Citrus species and cultivars based on the chromomycin $\mathrm{A}_{3}$ (CMA) banding patterns. Journal of the Japanese Society for Horticultural Science, 70(1), 83-88. https://doi.org/10.2503/jjshs.70.83

Befu, M., Kitajima, A., \& Hasegawa, K. (2002). Classification of the Citrus chromosomes with same types of chromomycin a banding patterns. Journal of the Japanese Society for Horticultural Science, 71(3), 394-400. https://doi.org/10.2503/jjshs.71.394

Befu, M., Kitajima, A., Ling, Y. X., \& Hasegawa, K. (2000). Classification of 'Tosa-Buntan' pummelo (Citrus grandis [L.] Osb.), 'Washington' navel orange (C. sinensis [L.] Osb.) and trifoliate orange (Poncirus trifoliata [L.] Raf.) chromosomes using young leaves. Journal of the Japanese Society for Horticultural Science, 69(1), 22-28. https://doi.org/10.2503/jjshs.69.22

Brasileiro-Vidal, A. C., Santos-Serejo, J. A., Soares Filho, W. S., \& Guerra, M. (2007). A simple chromosomal marker can reliably distinguish Poncirus from Citrus species. Genetica, 129(3), 273-279. https://doi.org/ 10.1007/s10709-006-0007-4

Carbonell-Caballero, J., Alonso, R., Ibañez, V., Terol, J., Talon, M., \& Dopazo, J. (2015). A Phylogenetic Analysis of 34 Chloroplast genomes elucidates the relationships between wild and domestic species within the genus Citrus. Molecular Biology and Evolution, 32(8), 2015-2035. https://oi.org/10.1093/ molbev/msv082

Carvalho, R., Soares Filho, W.S., Brasileiro-Vidal, A. C., \& Guerra, M. (2005). The relationship among lemons, limes and citron: The chromosomal comparison. Cytogenetic and Genome Research, 109(1-3), $276-282$. https://doi.org/10.1159/000082410

Cornélio, M. T. M. N., Figueirôa, A. R. S., Santos, K. G. B., Carvalho, R., Soares Filho, W. S., \& Guerra, M. (2003). Chromosomal relationships among cultivars of Citrus reticulata Blanco, its hybrids and related species. Plant Systematics and Evolution, 240(1-4), 149-161. https://doi.org/10.1007/s00606-003-0012-3

Da Costa Silva, S. C., Marques, A., Soares Filho, W. S., Mirkov, T. E., Pedrosa-Harand, A., \& Guerra, M. (2011). The cytogenetic map of the Poncirus trifoliata (L.) Raf.- The nomenclature system for all chromosomes of citric species. Tropical Plant Biology, 4(2), 99-105. https://doi.org/10.1007/s12042-011-9072-7

Da Costa Silva, S. C., Mendes, S., Soares Filho, W. S., \& Pedrosa-Harand, A. (2015). Chromosome homologies between Citrus and Poncirus - The comparative cytogenetic map of mandarin (Citrus reticulata). Tree Genetics and Genomes, 11(1), 1-9. https://doi.org/10.1007/s11295-014-0811-4

Fonsêca, A., Ferreira, J., dos Santos, T. R. B., Mosiolek, M., Bellucci, E., Kami, J., ... Pedrosa-Harand, A. (2010). Cytogenetic map of common bean (Phaseolus vulgaris L.). Chromosome Research, 18(4), 487-502. https://doi.org/10.1007/s10577-010-9129-8

Froelicher, Y., Mouhaya, W., Bassene, J. B., Costantino, G., Kamiri, M., Luro, F., ... Ollitrault, P. (2011). New Universal PCR mitochondrial markers reveal new information on maternal citrus phylogeny. Tree Genetics and Genomes, 7(1), 49-61. https://doi.org/10.1007/s11295-010-0314-x

Garcia-Lor, A., Curk, F., Snoussi-Trifa, H., Morillon, R., Ancillo, G., Luro, F., ... Ollitrault, P. (2013). A nuclear phylogenetic analysis: SNPs, indels and SSRs deliver new insights into the relationships in the "true citrus fruit trees' group (Citrinae, Rutaceae) and the origin of cultivated species. Annals of Botany, 111(1), 1-19. https://doi.org/10.1093/aob/mcs227

Green, R. M., Vardi, A., \& Galun, E. (1986). The plastome of Citrus. Physical map, variation among Citrus cultivars and species, and comparison with related genera. Theoretical and Applied Genetics, 72(2), 170-177. https://doi.org/10.1007/BF00266989 
Guerra, M. (1986). Reviewing the chromosome nomenclature of Levan et al. Brazilian Journal of Genetics, 9(4), 741-743.

Guerra, M. (1993). Cytogenetics of Rutaceae. V. High chromosomal variability in Citrus species revealed by CMA/DAPI staining. Heredity, 71(3), 234-241. https://doi.org/10.1038/hdy.1993.131

Guerra, M. (2009). Chromosomal variability and the origin of Citrus species. In C. Mahoney L., \& D. A. Springer (Eds), Genetic Diversity (pp. 51-68). Nova Science Publishers Inc., New York, USA.

Heslop-Harrison, J. S., Harrison, G. E., \& Leitch, I. J. (1992). Reprobing of DNA: DNA hybridization in situ preparations. Trends in Genetics, 8(11), 372-373. https://doi.org/10.1016/0168-9525(92)90287-E

Kayum, M., Koç, N. K., \& Rokka, V. M. (1998). Variation of the nuclear DNA content of species of subtribe Citrinae (Rutaceae). HortScience, 33(7), 1247-1250. https://doi.org/10.21273/HORTSCI.33.7.1247

Lan, H., Chen, C. L., Miao, Y., Yu, C. X., Guo, W. W., Xu, Q., \& Deng, X. X. (2016). Fragile sites of 'Valencia' sweet orange (Citrus sinensis) chromosomes are related with active 45S rDNA. PLoS One, 11(3), e0151512. https://doi.org/10.1371/journal.pone.0151512

Li, X., Xie, R., Lu, Z., \& Zhou, Z. (2010). The origin of the cultivated Citrus inferred from internal transcribed spacer and chloroplast DNA sequence and amplified fragment length polymorphism fingerprints. Journal of the American Society for Horticultural Science, 135(4), 341-350. https://doi.org/10.21273/JASHS. 135.4.341

Matsuyama, T., Akihama, T., Ito, Y., Fukui, M., \& Omura, K. (1996). Characterization of heterochromatic regions in 'Trovita' orange (Citrus sinensis, Osbeck) chromosomes by the staining and FISH fluorescent methods. Genome, 39(5), 941-945. https://doi.org/10.1139/g96-118

Mendes, S., Moraes, A. P., Mirkov, T. E., \& Pedrosa-Harand, A. (2011). Chromosome homeologies and high variation in heterochromatin distribution between Citrus L. and Poncirus Raf. the evidenced by comparative cytogenetic mapping. Chromosome Research, 19(4), 521-530. https://doi.org/10.1007/s10577-011-9203-x

Miranda, M., Ikeda, M., Endo, T., Moriguchi, T., \& Omura, M. (1997). Comparative analysis on the distribution of heterochromatin in Citrus, Poncirus and Fortunella chromosomes. Chromosome Research, 5(2), 86-92. https://doi.org/10.1023/A:1018409922843

Moore, G. A. (2001). Oranges and lemons: Clues to the taxonomy of Citrus from molecular markers. Trends in Genetics, 17(9), 536-540. https://doi.org/10.1016/S0168-9525(01)02442-8

Moraes, A. P., Lemos, R. R., Brasileiro-Vidal, A. C., Soares Filho, W. S., \& Guerra, M. (2007a). Chromosomal markers distinguish hybrids and non-hybrid accessions of mandarin. Cytogenetic and Genome Research, 119(3-4), 275-281. https://doi.org/10.1159/000112074

Moraes, A. P., Mirkov, T. E., \& Guerra, M. (2008). Mapping the chromosomes of Poncirus trifoliata Raf. by BAC-FISH. Cytogenetic and Genome Research, 121(3-4), 277-281. https://doi.org/10.1159/000138897

Moraes, A. P., Soares Filho, W. S., \& Guerra, M. (2007b). Karyotype diversity and the origin of grapefruit. Chromosome Research, 15(1), 115-121. https://doi.org/10.1007/s10577-006-1101-2

Nicolosi, E., Deng, Z. N., Gentile, A., La Malfa, S., Continella, G., \& Tribulato, E. (2000). Citrus phylogeny and genetic origin of the important species investigated by molecular markers. Theoretical and Applied Genetics, 100(8), 1155-1166. https://doi.org/10.1007/s001220051419

Ollitrault, P., Dambier, D., Luro, F., \& Duperray, C. (1994). Nuclear genome size variations in Citrus. Fruits, 49, 390-393.

Pang, X. M., Hu, C. G., \& Deng, X. X. (2007). Phylogenetic relationship within Citrus and related genera inferred from the AFLP markers. Genetic Resources and Crop Evolution, 54(2), 429-436. https://doi.org/ $10.1007 / \mathrm{s} 10722-006-0005-5$

Pedrosa, A., Sandal, N., Stougaard, J., Schweizer, D., \& Bachmair, A. (2002). Chromosomal map of the model legume Lotus japonicus. Genetics, 161(4), 1661-1672.

Pedrosa, A., Schweizer, D., \& Guerra, M. (2000). Cytological heterozygosity and the hybrid origin of sweet orange [Citrus sinensis (L.) Osbeck]. Theoretical and Applied Genetics, 100(3-4), 361-367. https://doi.org/ $10.1007 / \mathrm{s} 001220050047$ 
Penjor, T., Anai, T., Nagano, Y., Matsumoto, R., \& Yamamoto, Y. (2010). Phylogenetic relationships of Citrus and its relatives based on $r b c L$ gene sequences. Tree Genetics \& Genomes, 6(6), 931-939. https://doi.org/ 10.1007/s11295-010-0302-1

Penjor, T., Yamamoto, M., Uehara, M., Ide, M., Matsumoto, N., Matsumoto, R., \& Nagano, Y. (2013). Phylogenetic relationships of Citrus and its relatives based on matK gene sequences. PLoS One, 8(4), e62574. https://doi.org/10.1371/journal.pone.0062574

Scora, R. W. (1975). IX. On the history and origin of Citrus. Bull Torrey Botanical Club, 102(6), 369-375. https://doi.org/10.2307/2484763

Swingle, W. T., \& Reece, P. C. (1967). The botany of Citrus and its wild relatives. In W. Reuther, H. J. Weber, \& L. D. Batchelor (Eds.), The Citrus industry. History, world distribution, botany and varieties (Vol. I, pp. 190-430). University of California Press, Berkeley, USA.

Uzun, A., Yesiloglu, T., Aka-Kacar, Y., Tuzcu, O., \& Gulsen, O. (2009). Genetic diversity and relationships within Citrus and related genera based on sequence related amplified polymorphism markers (SRAPs). Scientia Horticulturae, 121(3), 306-312. https://doi.org/10.1016/j.scienta.2009.02.018

Velasco, R., \& Licciardello, C. (2014). A genealogy of the citrus family. Nature Biotechnology, 32(7), 640-642. https://doi.org/10.1038/nbt.2954

Wang, X., Xu, Y., Zhang, S., Cao, L., Huang, Y., Cheng, J., ... Xu, Q. (2017). Genomic analyses of primitive, wild and cultivated citrus provide insights into asexual reproduction. Nature Genetics, 49(5), 765-772. https://doi.org/10.1038/ng.3839

Wu, G. A., Prochnik, S., Jenkins, J., Salse, J., Hellsten, U., Murat, F., ... Rokhsar, D. (2014). Sequencing of diverse mandarin, pummelo and orange genomes reveals complex history of admixture during citrus domestication. Nature Biotechnology, 32(7), 656-662. https://doi.org/10.1038/nbt.2906

Wu, G. A., Terol, J., Ibanez, V., López-García, A., Pérez-Román, E., Borredá, C., ... Talon, M. (2018). Genomics of the origin and evolution of Citrus. Nature, 554(7692), 311-316. https://doi.org/10.1038/nature25447

Xu, Q., Chen, L. L., Ruan, X., Chen, D., Zhu, A., Chen, C., ... Ruan, Y. (2013). The draft genome of sweet orange (Citrus sinensis). Nature Genetics, 45(1), 59-66. https://doi.org/10.1038/ng.2472

Yamamoto, M., Abkenar, A. A., Matsumoto, R., Nesumi, H., Yoshida, T., Kuniga, T., ... Tominaga, S. (2007). CMA banding patterns of chromosomes in major citrus species. Journal of the Japanese Society for Horticultural Science, 76(1), 36-40. https://doi.org/10.2503/jjshs.76.36

Yamamoto, M., Kobayashi, S., Nakamura, Y., \& Yamada, Y. (1993). Phylogenic relationships of citrus revealed by RFLP analysis of mitochondrial and chloroplast DNA. Japanese Journal of Breeding, 43(3), 355-365. https://doi.org/10.1270/jsbbs1951.43.355

Yang, X., Kitajima, A., \& Hasegawa, K. (2002). Chromosome pairing set and the presence of unreduced gametes explain the possible origin of polyploid progenies from the diploids 'Tosa-Butan' $\times$ 'Suisho-Butan' pummelo. Journal of the Japanese Society for Horticultural Science, 71(4), 538-543. https://doi.org/ $10.2503 /$ jjshs. 71.538

Yang, Z. N., Ye, X. R., Choi, S., Molina, J., Moonan, F., Wing, R. A., ... Mirkov, T. E. (2001). Construction of the $1.2 \mathrm{Mb}$ contig including the citrus tristeza virus resistance gene locus using the bacterial artificial chromosome library of Poncirus trifoliata (L.) Raf. Genome, 44(3), 382-393. https://doi.org/10.1139/ gen-44-3-382 


\section{Appendix A}

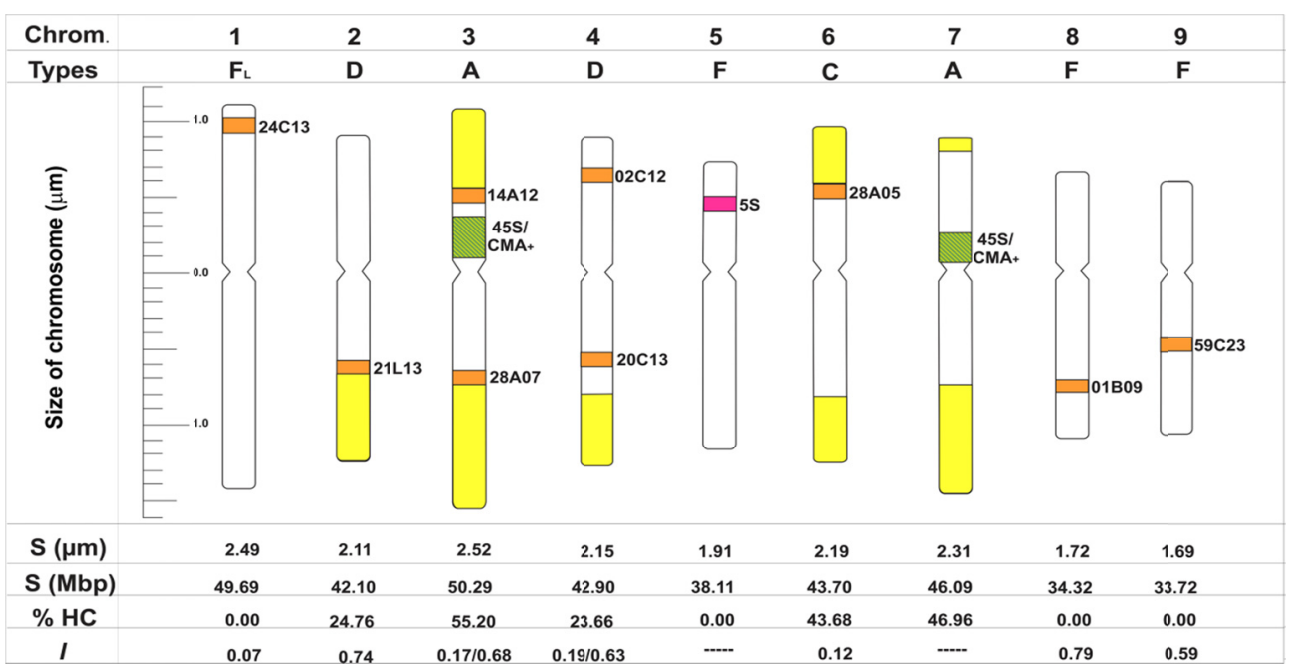

Figure A1. Idiogram of Citrus maxima (Burm.) Merril 'Pink' showing chromosome types, individual average size of chromosomes (S) in $\mu \mathrm{m}$ and Mbp, percentage of heterochromatin per chromosome (\% HC) and the mean relative location of BAC clones (1, with telomere of short arm being 0 and telomere of long arm, 1). When two BACs were located on the same chromosome (chromosomes 3 and 4 ), the first value correspond to the location of the BAC from the short arm and the second value to the BAC from the long arm. The 35S rDNA sites are shown in green and yellow, in case they were co-localized with the CMA positive bands (Moraes et al., 2007b), whereas the $5 \mathrm{~S}$ rDNA is shown in red. The CMA positive bands and position of BAC clones are shown in yellow and orange, respectively
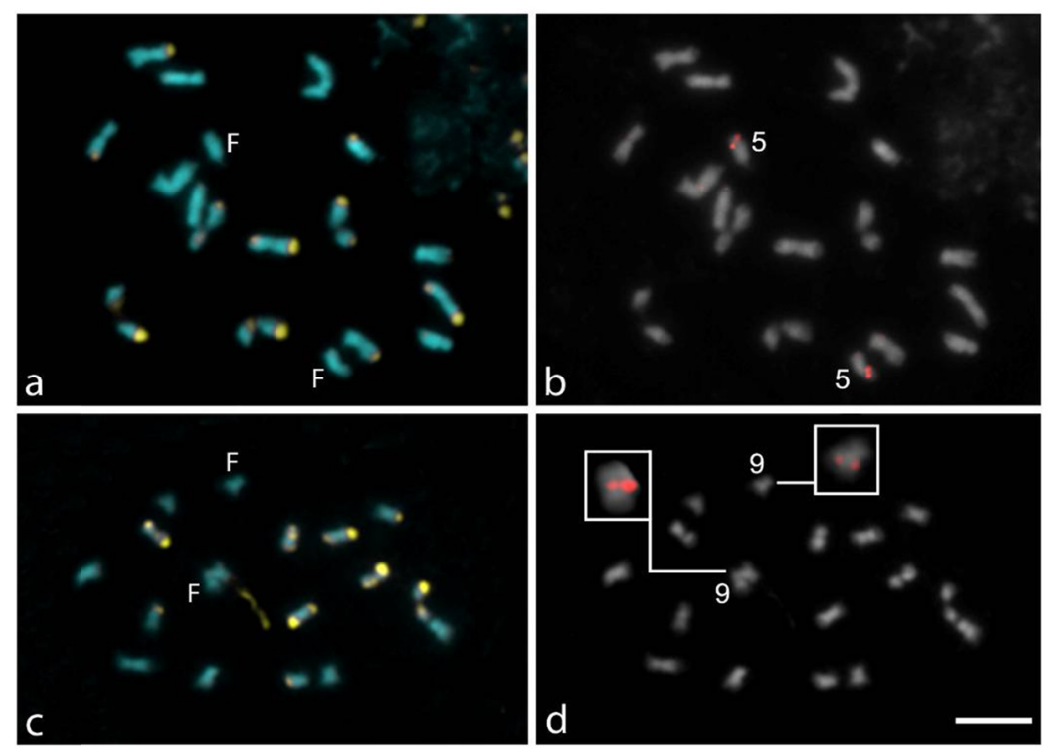

Figure A2. Metaphase cells from Citrus maxima (Burm.) Merril 'Pink' stained with CMA/DAPI (a) and (c) and in situ hybridization with the 5S rDNA (chr. 5) (b) and BAC 59C23 (chr. 9) (d). Insets in (d) show enlarged chromosomes with greater contrast and magnification. Bar in (d) corresponds to 2.5 micrometers

\section{Copyrights}

Copyright for this article is retained by the author(s), with first publication rights granted to the journal.

This is an open-access article distributed under the terms and conditions of the Creative Commons Attribution license (http://creativecommons.org/licenses/by/4.0/). 